\title{
1 Hybridisation boosts dispersal of two contrasted ecotypes in a grass species
}

2

3 Emma V. Curran ${ }^{1,4}$, Matilda S. Scott ${ }^{1}$, Jill K. Olofsson ${ }^{1,5}$, Florence Nyirenda ${ }^{2}$, Graciela

4 Sotelo $^{1}$, Matheus E. Bianconi ${ }^{1}$, Sophie Manzi ${ }^{3}$, Guillaume Besnard ${ }^{3}$, Pascal-Antoine

5 Christin $^{1, *}$

6

$7 \quad{ }^{1}$ Department of Animal and Plant Sciences, University of Sheffield, Sheffield S10 2TN,

8 United Kingdom

$9 \quad{ }^{2}$ Department of Biological Sciences, University of Zambia, Lusaka, Zambia

$10{ }^{3}$ Laboratoire Evolution \& Diversité Biologique (EDB UMR5174), Université de Toulouse III

11 - Paul Sabatier, CNRS, IRD, 118 route de Narbonne, 31062 Toulouse, France

$12{ }^{4}$ Present address: School of Life Sciences, University of Nottingham, University Park,

13 Nottingham NG7 2RD, United Kingdom

$14{ }^{5}$ Present address: Section for GeoGenetics, Globe Institute University of Copenhagen, Øster

15 Voldgade 5-7, DK-1350 Copenhagen, Denmark

16 * Author for correspondence: p.christin@sheffield.ac.uk

17

18 Total word count: 6172 words

19 Introduction: 617 words

20 Material and Methods: 2053 words

21 Results: 1865 words

22 Discussion: 1437 words

23 This manuscript contains five figures, all in colour. 


\section{Summary}

44 Keywords: dispersal, ecology, gene flow, growth strategies, hybridisation, Poaceae

- In the absence of strong reproductive barriers, genetic exchanges between closely related groups of organisms with different adaptations have well-documented beneficial and detrimental consequences. In plants, pollen-mediated exchanges affect the sorting of alleles across physical landscapes, and influence rates of hybridisation. How these dynamics affect the emergence and spread of novel ecological strategies remains only partially understood.

- We use phylogenomics and population genomics to retrace the origin of two geographically overlapping ecotypes of the African grass Alloteropsis angusta.

- We report the existence of a previously undescribed ecotype inhabiting miombo woodlands and grasslands. The two ecotypes have divergent nuclear genomes. However, the seed-transported chloroplast genomes are consistently shared by distinct ecotypes inhabiting the same region. These patterns suggest that the nuclear genome of one ecotype can reach the seeds of the other via pollen movements, with strong selection subsequently sorting nuclear alleles by habitat.

- The contrasting ecotypes of $A$. angusta can use each other as a gateway to new locations across a large part of Africa. Coupled with newly discovered hybridisation with the sister species $A$. semialata, our results show that hybridisation can facilitate the geographical dispersal of distinct ecotypes of the same grass species.

3 


\section{Introduction}

46 When populations encounter new environments, the selection pressures they face may initiate

47 the evolution of novel adaptive phenotypes, enabling them to colonise previously untapped

48 niches. Contrasting conditions between novel and ancestral habitats can impose divergent

49 selection on populations, resulting in reproductively isolated ecotypes within a species

50 (Schluter, 2001). Such ecotypes may represent early stages of the 'speciation continuum'

51 (Seehausen et al., 2014), and, given time, they may become fully isolated species. Prior to

52 complete reproductive isolation, however, hybridisation between diverged ecotypes is

53 commonplace (e.g. in monkeyflowers: Stankowski et al., 2017; marine snails: Galindo et al.,

54 2013; and whitefish: Lu et al., 2001). Hybridisation between geographically isolated

55 populations may result in a breakdown of differentiation, leading to loss of local adaptation

56 and the formation of homogenised 'hybrid swarms' (Taylor et al., 2006; Abbott et al., 2013).

57 Hybridisation can also contribute to the overall success of populations. Adaptive genetic

58 material may be shared between populations via introgression, conferring advantageous traits

59 (e.g. Song et al., 2011; The Heliconius Genome Consortium, 2012; Arnold et al., 2016). In

60 sessile organisms, such as plants, hybridisation can affect the spatial movements of genes

61 (Martinsen et al., 2001; Petit et al., 2003). Plant pollen can be exchanged over long distances,

62 resulting in hybridisation with both positive and negative consequences (Ellstrand, 1992,

63 2014). Indeed, long-distance cross pollination can restore diversity, but also lead to the

64 swamping and extinction of rare types (Ellstrand, 1992; Aguilée, et al., 2016; Hall, 2016;

65 Todesco et al., 2016). However, the dynamics that govern barriers to gene flow within plant

66 species, i.e. the balance between gene flow and divergent selection (Barton \& Hewitt, 1985),

67 and the consequences of recurrent hybridisation are still not fully understood. 
69 of their growth plan and variation in life strategies (Linder et al., 2018). They range from

70 short annuals to large, tree-like bamboos, and include mat-forming alpine species, aquatic

71 plants, and decumbent species crawling over large areas (Grass Phylogeny Working Group,

72 2001; Perreta et al., 2011; Kellogg, 2015). However, the evolutionary dynamics underlying

73 the origins of different growth forms remain poorly studied. The grass genus Alloteropsis is

74 composed of five recognised species (Clayton and Renvoize 1982; Ibrahim et al., 2008). The

75 two sister species A. semialata and A. angusta are perennials. The former inhabits the

76 grasslands and savannah woodlands of Africa, Asia and Oceania (Lundgren et al., 2015), and

77 its stems grow erect from bulb-like structures formed by thickened sheaths (Clayton and

78 Renvoize 1982). The plant propagates vegetatively via very short rhizomes that lead to a

79 proliferation of bulbs next to each other. By contrast, A. angusta is reported as a slender,

80 decumbent species growing in swamps, and is only known from Central and East Africa

81 (Stapf 1919; Clayton and Renvoize 1982). While studying the origins of novel photosynthetic

82 types in A. semialata, we discovered some specimens assigned to this species that, based on

83 both organelle and nuclear genomes, corresponded to A. angusta (e.g. sample "Pauwels 1182

84 [BRU]”; Olofsson et al., 2016). These misidentified samples were erect, with bulb-like

85 enlargements at the base of the stem, showing that $A$. angusta can occur as solid erect plants

86 that resemble $A$. semialata in addition to the previously reported fragile decumbent type.

87 Because the growth forms observed in the field persisted when plants were grown in

88 controlled conditions at the University of Sheffield, they must be genetically determined.

89 Alloteropsis angusta therefore constitutes an outstanding system to study the evolutionary

90 dynamics leading to habitat specialisation of the growth form within grass species. Since this 
91 species has not yet been the focus of dedicated studies, the status of the two morphs and their

92 relationship remain unknown.

In this study, we combine phylogenomics and populations genomics to study the

94 dynamics underlying the functional diversification of A. angusta in Africa. We quantify the

95 morphological variation within $A$. angusta to (i) confirm the existence of two morphs

96 associated with different environments. We then sequence the genomes of 13 individuals of

97 A. angusta and infer the phylogenetic tree of chloroplast genomes to (ii) determine whether

98 maternally-inherited genomes of each morph spread independently. Phylogenetic analyses of

99 the nuclear genomes from the same individuals are then used to (iii) infer the relationships

100 among the two morphs. We scan the genomes of numerous populations spread from Zambia

101 to Uganda and use population genomics to (iv) test for frequent admixture between the two

102 types and assess the geographical patterns of genetic variation. Finally, ABBA-BABA tests

103 are conducted to (v) test for introgression between the two morphs and with the sister species

104 A. semialata. Our results support the importance of frequent hybridisation among the two

105 morphs of A. angusta for the dispersal of this species, despite a strong ecological barrier. We

106 also reveal episodic exchanges between $A$. semialata and one of the morphs of $A$. angusta,

107 suggesting that the two species might form a loose species complex.

\section{Materials and Methods}

\section{Population sampling}

111 Samples of Alloteropsis angusta were obtained from herbaria, or from fieldwork conducted

112 in Uganda, Tanzania and Zambia (Table S1; Olofsson et al., 2016; Dunning et al., 2017).

113 Denser, population-level sampling was conducted in Zambia through walk-and-search stops

114 in areas covered by miombo woodlands, grasslands or swamps (Table S1). For each 
115 population found, GPS coordinates were recorded with a description of the habitat, and up to

116 ten distinct individuals, growing at least one metre apart, were collected in silica gel. For

117 most populations, several individuals were pressed and later used to prepare herbarium

118 vouchers (listed in Table S1). When A. angusta and A. semialata grew together, both were

119 sampled. In addition, we sampled 46 plants with GPS coordinates for each individual in a

120 location where contrasted morphs were observed (population ZAM1930).

123 The 80 available herbarium vouchers were digitised and measured with ImageJ (Schneider et

124 al., 2012; Table S2). To capture variation in vegetative organs, we measured the size of the

125 bulb, stems and leaves from all samples. The bulb length was measured from its bottom

126 extremity to the limit between protective sheaths and rosette leaves, while the bulb width was

127 measured as the largest width perpendicular to the growth axis. The stem length was

128 measured from the limit of the bulb to the split of the racemes, and its width was measured at

129 the base, just above the bulb, and at the top, just below the racemes. For leaves, the length of

130 the lamina of the longest rosette leaf and longest stem leaf were measured. To capture

131 reproductive characters, the length of the longest raceme was measured, together with the

132 average length of ten spikelets and the average spacing between all consecutive spikelets

133 along a raceme. The anatomical variation was summarised with a principal component

134 analysis (PCA), considering all characters except the spikelet length, which could not be

135 typed on individuals that lost their florets before collection. The PCA was performed using

136 the prcomp function in R version 3.6.0 (R Core Team, 2019). 
139 Genome sequencing and phylogenetic analysis of chloroplast genomes

140 Phylogenetic relationships within and between the two sister species were inferred using

141 whole genome re-sequencing data. The genomes of two herbarium samples and three field-

142 collected samples were sequenced using a genome skimming approach, with 150-bp paired-

143 end Illumina reads, as described in Olofsson et al. (2016). In addition, four field-collected

144 individuals representing a diversity of geographic origins and growth habits were sequenced

145 as 250-bp paired-end reads, as described in Dunning et al. (2019) (Table S1). Other genome

146 datasets of $A$. angusta, A. semialata and A. cimicina were retrieved from previous work

147 (Table S1).

148 Complete chloroplast genomes were assembled using the genome walking approach

149 described in Lundgren et al., 2015. Assembled plastomes were aligned using ClustalW, the

150 second repeat was removed, and the alignment was manually refined. The final alignment

151 was 120,271 bp long, and a time-calibrated phylogenetic was inferred with Beast v. 1.8.4

152 (Drummond and Rambaut, 2007). A log-normal relaxed clock was used, with a GTR+G

153 substitution model and a constant-size coalescent prior. The monophyly of the ingroup (all

154 samples other than the outgroup A. cimcina) was enforced to root the tree. The root of the tree

155 was set to $11.46 \mathrm{Ma}$ and the split of A. angusta and A. semialata to 8.075 (using a normal

156 distribution of 0.0001), following previous estimates (Lundgren et al., 2015). Two analyses

157 were run for 50,000,000 generations, sampling a tree every 10,000 generations. Convergence

158 of the runs was monitored using Tracer v. 1.6.0 (Rambaut et al., 2013), and the burn-in

159 period was set to $10,000,000$ generations. The median ages of posterior trees were mapped on 160 the maximum credibility tree. 
163 Phylogenetic analyses of nuclear genomes

164 Nuclear variants were extracted from sequencing reads of whole-genome sequenced samples.

165 Raw reads obtained from fresh samples were cleaned using NGS QC Toolkit v. 2.3.3 (Patel

166 \& Jain, 2012) to remove reads where more than $20 \%$ of the bases had a quality score below

167 Q20, and those which had ambiguous bases. Bases with quality score below Q20 were further

168 trimmed from the 3' end of reads. Adaptors were removed using NxTrim (O’Connell et al.,

169 2015). Raw reads from low coverage sequenced individuals were cleaned as in Olofsson et

170 al. (2016). All cleaned reads were aligned to the $A$. semialata reference genome

171 (ASEM_AUS1_v1.0; GenBank accession QPGU01000000; Dunning et al., 2019) using

172 Bowtie2 v. 2.3.5.1 (Langmead \& Salzberg, 2012) with the default settings for paired-end

173 reads. Read alignment files were cleaned, sorted and indexed using samtools v.1.9 (Li, 2011).

174 PCR duplicates were removed using Picard Tools v.1.102 (Broad Institute, 2019).

175 For each sample, reads mapped to the reference nuclear genome were separated out

176 from those mapped to the reference mitochondrial or chloroplast genomes. A multi-sample

177 sequence alignment was then generated across all individuals. A consensus sequence was

178 generated from the reference-mapped reads for each individual using the mpileup tool in

179 samtools v.1.9 (Li, 2011), and a custom bash script was then used to filter for depth the

180 consensus sequences of re-sequenced individuals (Olofsson et al., 2016), removing bases

181 supported by less than three reads. The genome-skimmed individuals were not filtered for

182 depth, due to the low average coverage for these samples. Polymorphic sites were called as

183 ambiguous bases following IUPAC codes. Sites with more than $10 \%$ missing data were

184 removed using trimAl v. 1.4.rev6 (Capella-Gutiérrez et al., 2009), resulting in an alignment

185 of 142,848 bp. A maximum likelihood phylogeny was estimated under the GTR+CAT 
186 substitution model in RAxML v. 8.2.10 (Stamatakis, 2014) with 100 bootstrap

187 pseudoreplicates.

188 We also estimated a multigene coalescent phylogeny from the nuclear genomes. For

189 this, we first retrieved a dataset consisting of 7,408 putative single-copy orthologs of

190 Panicoideae grasses (the tribe including Alloteropsis) from a previous study (Bianconi et al.,

191 2020), and the corresponding coding sequences of $A$. semialata were used here as references

192 for the assembly of gene sequences, as described for the whole genome. The resulting gene

193 alignments were trimmed using trimAl to remove sites with more than $30 \%$ missing data, and

194 sequences shorter than 200 bp after trimming were discarded. Finally, only gene alignments

195 longer than 500 bp and with more than 95\% of taxon occupancy were retained for

196 phylogenetic analyses. A maximum-likelihood phylogeny was estimated for each of the

197 remaining 2,960 alignments using RAxML, with a GTR+CAT substitution model and 100

198 bootstrap pseudoreplicates. Gene trees were then summarised into a multigene coalescent

199 phylogeny using Astral v5.5.9 (Zhang et al., 2018) after collapsing branches with bootstrap

200 support below 50.

202 Population-level genetic structure within A. angusta

203 The genomes of population-level samples were scanned using a reduced representation

204 approach, restriction site associated DNA (RAD) sequencing, as described in Olofsson et al.

205 (2019). In brief, the DNA of up to five individuals of each population (more in ZAM1930

206 where the two morphs occurred) were double digested and then pooled before sequencing 72

207 - 96 individuals per lane of Illumina HiSeq 2500 at the Sheffield Diagnostic Genetics

208 Service, Sheffield Children's NHS Foundation Trust. Our final dataset included 196 A. 
angusta individuals, and 15 newly sequenced $A$. semialata, which were combined with 49

210 previously sequenced $A$. semialata (Olofsson et al., 2021; Table S1).

211 We used a PCA of genetic variation and individual ancestry analyses to describe the

212 distribution of genetic diversity among these populations. The program Trimmomatic (Bolger

213 et al., 2014) was used to trim raw RAD-sequencing reads to remove adaptor and other

214 Illumina-specific sequences and bases with a low quality score $(\mathrm{Q}<3)$ from the 5' and 3'

215 ends. Reads were further clipped when the average quality within a sliding window of four

216 bases dropped below an average quality threshold $(\mathrm{Q}<15)$. The pooled reads were de-

217 multiplexed into individually barcoded samples using the module 'process_radtags' in the

218 program STACKS (Catchen et al., 2013). Reads were then mapped to the A. semialata

219 reference genome (ASEM_AUS1_v1.0; GenBank accession QPGU01000000; Dunning et

220 al., 2019), using Bowtie2 with the default settings for paired-end reads. We estimated

221 genotype likelihoods from the reference-mapped reads for each individual, using the program

222 ANGSD (Korneliussen et al., 2014). Sites present in at least 50\% of the individuals with a

223 minimum depth of 5 per individual and with minimum mapping and base quality scores of 20

224 were included in the analysis. Individuals with more than 99\% missing data were removed.

225 After filtering, 49,523 sites remained, across 196 individuals. The proportion of each

226 individual's genome that can be assigned to a specified number of genetic clusters $(K)$ was

227 estimated from the genotype likelihoods using the software NGSadmix (Skotte et al., 2013).

228 NGSadmix was run with $K$ ranging from 1 to 10 , with five replicates for each run, each with

229 a random starting seed. The explanatory power of the increasing $K$ values was assessed using

230 the $\Delta K$ criterion (Evanno et al., 2005), implemented with the CLUMPAK tool (Kopelman et

231 al., 2015). A PCA was carried out using PCAngsd (Meisner \& Albrechtsen, 2018), which 
254 To test for gene flow among A. angusta lineages and between A. angusta and A. semialata, 255 we used the ABBA-BABA method (Durand et al., 2011; Green et al., 2010). This approach

estimates a covariance matrix using the genotype likelihoods. We retrieved the principal components of genetic structure using eigenvector decomposition in R.

To establish the genetic structure within and between the erect and decumbent morphs of $A$. angusta, we calculated pairwise genome-wide $F_{\mathrm{ST}}$ between all A. angusta populations using Hudson’s $F_{\text {ST }}$ estimator (Hudson et al., 1992). This was calculated for each SNP based on allele frequencies estimated directly from the data, as in Soria-Carrasco et al. (2014). Average genome-wide $F_{\mathrm{ST}}$ was calculated as a ratio of averages, by averaging the within- and between-population variance components separately as recommended by Bhatia et al. (2013), using an R script by Soria-Carrasco (2019: https://github.com/visoca/popgenomworkshophmm). The erect and decumbent individuals of population ZAM1930 were considered as two distinct populations for these analyses. Pairwise geographic distances between pairs of populations were calculated using the 'rdist.earth' function in the R package ‘fields' (Nychka et al., 2017). A relationship between genetic and geographic distances was tested using Mantel tests, with 9,999 permutations, for populations within each of the erect and decumbent groups, and among them. In each case, the strength of the relationship was assessed with Spearman's correlation coefficient $(\rho)$. To examine the genome-wide landscape of genetic differentiation between the decumbent and erect morphs, we used the $F_{\text {ST }}$ calculated for each SNP between them in population ZAM1930, for which there are 20 decumbent and 26 erect individuals. Average $F_{\text {ST }}$ was calculated, as above, for non-overlapping sliding windows of 500kb.

\section{Tests for gene flow between morphological types of A. angusta and between species}


uses patterns of ancestral and derived alleles to calculate $D$-statistics and test for asymmetry

257 in the frequencies of phylogenies incongruent with the species tree beyond what is expected

258 under a scenario of incomplete lineage sorting (Durand et al., 2011; Green et al., 2010).

259 Three ingroups (P1, P2, P3) and one outgroup (O) are required, in the configuration (((P1,

260 P2), P3), O), and here A. cimicina was used as the outgroup. Tests were carried out using the

261 -doAbbababa option in the program ANGSD (Korneliussen et al., 2014), to compute the $D$

262 statistic. Deviation from the null expectation $(D=0)$ was tested using the jackKnife.R script

263 (block jackknife method) provided with ANGSD.

To test for gene flow among A. angusta lineages, we considered first all combinations

265 where P1 and P2 are occupied by a decumbent individual and P3 by an erect individual, and

266 then all combinations where P1 and P2 are occupied by an erect individual and P3 is

267 occupied by a decumbent individual. The Bonferroni correction was used to adjust p-values

268 with the total number of such combinations, but only a subset was subsequently considered to

269 simplify the dataset. For each of the two types of combinations, the individual most often

270 found as the least introgressed was identified and then fixed in the P1 position, so that all

271 positive $D$-statistics indicate gene flow between P2 and P3. A similar approach was used to

272 test for gene flow between $A$. angusta and A. semialata, considering all combinations where

273 P1 and P2 are occupied by A. angusta individuals and P3 is an individual from A. semialata.

274 Again, p-values were corrected for the number of such comparisons, but only those with the

275 least often introgressed individual in the P1 position were considered. 


\section{Results}

281 Two growth forms associated with distinct habitats

282 We collected six populations of A. angusta in 2015 in Uganda, in boggy areas around lakes or 283 other water soaked grasslands. All these individuals were decumbent, with long fragile stems

284 crawling among other grasses. The stems were branching, with secondary roots growing from 285 nodes (e.g. Fig. 1C). Individuals brought back as live cuttings have been living in controlled 286 conditions for > six years, with the same growth habit. They spread horizontally by making 287 new roots from crawling stems that can then produce new individuals. Using the approach 288 described in Bianconi et al. (2020), we estimated the genome size for two decumbent 289 individuals, and the estimated values were similar to diploids of the sister species $A$. 290 semialata (individual MRL15-04-06, 2C = 2.09 Gb; MRL15-04-08, 2C = 1.95 Gb). The

291 individuals of A. angusta collected in Tanzania in 2016 and Zambia in 2017 were clearly

292 distinct from the Ugandan ones. They grew in miombo woodlands and associated grasslands

293 from small bulbs, each with a single erect stem (e.g. Fig. 1B). We grew one individual from

294 seed in controlled conditions (from population ZAM1720), and it produced a bulb and grew 295 erect, as in the field. Its genome size was similar to that of Ugandan individuals $(2 \mathrm{C}=2.10$ 296 Gb).

297 In total, we collected 31 populations of A. angusta in Zambia and Tanzania. In four of 298 these populations, the individuals were described as decumbent in the field, with strongly 299 branching stems, crawling amongst other species. All of these decumbent individuals, which 300 were morphologically similar to those collected previously in Uganda, were found in water301 logged wetlands on the shores of rivers or lakes (Table S1; Fig. S1). Individuals from 26 of 302 the other populations were described in the field as erect. They formed bulb-like structures of 303 various sizes at the base of their stems and grew tall, with a single stem per bulb. Short 
304 rhizomes connected underground successive bulbs. These individuals match the description

305 of the defunct species A. gwebiensis (Stent \& Rattray, 1933), which was later merged with A.

306 semialata (Clayton \& Renvoize, 1982). These erect individuals were all found in miombo

307 woodlands and associated grasslands, with between 0 and 90\% tree cover (Table S1; Fig. S1).

308 While two of these erect populations were found in grasslands topping rivers, these

309 grasslands were not directly connected to the rivers and did not present the boggy

310 characteristics of the sites in which the decumbent forms were found. In seven of the erect

311 populations, A. angusta grew together with the conspecific A. semialata (Table S1). Finally,

312 population ZAM1930 spanned a miombo woodland sloping toward a river wetland.

313 Individuals growing in the miombo of this site were erect and found mixed with $A$.

314 semialata. By contrast, the individuals growing in the open river wetland were all decumbent 315 (Fig. S2).

317 The growth forms are morphologically distinct

318 The morphology of all sampled A. angusta, including herbarium vouchers, was analysed

319 together with a number of $A$. semialata (Table S2). The spikelet length of $A$. semialata ranged

320 from 4.28 to $6.32 \mathrm{~mm}$, while that of the decumbent $A$. angusta ranged from 2.7 to $3.75 \mathrm{~mm}$

321 (Table S2). These values correspond to the previously described A. semialata and A. angusta

322 (Clayton \& Renovize 1982), but the spikelet length of the erect $A$. angusta overlapped with

323 the two groups (3.13-4.52 mm; Table S2). A PCA confirmed that the erect and decumbent

324 forms of $A$. angusta occupy different parts of the morphological space (Fig. 1D), although the

325 growth habit was not included as a character. Important variation was observed within the

326 erect A. angusta, which overlap in the anatomical space with A. semialata (Fig. 1D).

327 Populations classified as decumbent occurred in a small subset of the anatomical space 
328 characterised by shorter and thinner bulbs, smaller rosette leaves, and thinner stems (Fig.

329 1D). The bulb width clearly discriminated the two types, being $0.90-2.57 \mathrm{~mm}$ in the

330 decumbent $A$. angusta and 3.06-12.81 $\mathrm{mm}$ in the erect ones (5.88-19.13 $\mathrm{mm}$ in A. semialata;

331 Table S2). The presence of bulbs is therefore a suitable character to define the two growth

332 habits of $A$. angusta, which are consistently associated with contrasted habitats and therefore 333 correspond to ecotypes (Figs 1, S1).

335 Chloroplast genomes are shared by the two ecotypes

336 The individuals selected for whole genome sequencing capture the morphological diversity

337 within the group (Fig. 1). The chloroplast phylogeny sorts A. angusta accessions by

338 geographic origin, independently of their ecotype (Figs 2B, S3). The first split separates two

339 groups, each with erect and decumbent individuals, and the decumbent accessions form four

340 distinct clades, while the erect accessions form five (Fig. 2B). In particular, the erect and

341 decumbent individuals from the West of Zambia (ZAM2074-14 and ZAM2075-04) are

342 grouped together (Fig. 2B). The two contrasted types from population ZAM1930 (ZAM1930-

343 JKO0102 and ZAM1930-17) are also grouped, with almost no chloroplast divergence (one

344 substitution, four 1-bp indels, and one 19-bp indel out of 117,652-bp pairwise alignment).

345 These patterns indicate that the history of the maternally-inherited chloroplasts is shared

346 between the erect and decumbent ecotypes. The divergence times among the plastomes of $A$.

347 angusta individuals are proportional to geographical distances (Mantel test, $\rho=0.67, P<$

348 0.001), indicating that the extant plastome diversity results from a gradual expansion. The

349 relationship remains significant when considering only pairs composed of the two ecotypes

350 (Mantel test, $\rho=0.57, P<0.001$ ), and the patterns are similar for the different types (Fig. 351 S4). 
353 In stark contrast to the chloroplast phylogeny, the maximum likelihood nuclear phylogeny

354 using whole genome sequenced individuals revealed that the erect and decumbent ecotypes

355 each form a distinct monophyletic group that covers the studied geographical region (Figs

356 2C, S3). Individuals of contrasting ecotypes that were collected from the same locality in

357 Zambia (population ZAM1930) group with their respective ecotype despite close geographic

358 proximity, with the decumbent individual (ZAM1930-17) showing a closer phylogenetic

359 relationship to decumbent individuals from Uganda (UGA1 and UGA4) than to erect

360 individuals growing a few metres away (ZAM1930-JKO0102).

361 The multigene coalescent species tree recovered the same relationships (Figs 2D, S5).

362 The monophyly of $A$. angusta was supported by almost all tree quartets, indicating lineage

363 sorting on the branch leading to A. angusta. By contrast, many quartet trees supported the two

364 topologies alternative to the main one for nodes leading to the erect A. angusta (Fig. 2D),

365 which indicates that high levels of incomplete lineage sorting occurred within A. angusta.

366 The amount of quartets supporting alternative topologies was much smaller in nodes of the

367 decumbent group (Fig. 2D). Overall, these phylogenetic patterns suggest that the nuclear

368 genomes of the two ecotypes diverged before the spread of A. angusta across Africa.

370 Nuclear genetic groups are maintained despite close geographic proximity

371 The genetic structure of A. angusta populations spread across Zambia, Tanzania and Uganda

372 was deciphered using population-level RAD-sequencing data (Fig. 3A). The largest

373 component of genetic variation separates the erect and decumbent ecotypes along the first

374 axis (23.9\% of the total variation, Fig. 3B). The second axis (9.71\% of the variation) splits

375 decumbent individuals according to geography, separating the more distant Ugandan 
376 populations from the rest of the individuals (Fig. 3B). The number of genetic clusters best

377 describing the population structure within A. angusta is two (Fig. S6), again sorting the

378 samples according to ecotype (Fig. 3C). Multiple decumbent individuals show suggestions of

379 introgression with the erect group (Fig. 3C), consistently across individuals of the decumbent

380 population ZAM2075 and the erect populations ZAM2074, ZAM2095, and ZAM2069.

381 Overall, across populations spread from Uganda to Zambia, the two ecotypes behave as

382 distinct nuclear groups (Fig. 3C). The structure remains strong between the ecotypes even

383 within the population where they grow adjacent to one another (ZAM19-30; Figs 3C, S2),

384 and again decumbent samples from this population are assigned to the same genetic cluster as

385 Ugandan samples growing $>1400 \mathrm{~km}$ away, instead of erect individuals growing a few meters

386 away. The nuclear patterns observed across the species range (Figs 2C, 2D) therefore

387 translate to smaller scales, with geographically close ecotypes associated with distinct and

388 divergent nuclear genomes.

Pairwise comparisons of genetic differentiation $\left(F_{\mathrm{ST}}\right)$ between populations reveal clear

390 isolation-by-distance within both the decumbent (Mantel test: $\rho=0.84, P<0.001$ ) and erect

391 (Mantel test: $\rho=0.43, P=0.0019$; Fig. 4) groups. However, the genetic divergence between

392 populations representing distinct ecotypes did not increase with geographic distance (Mantel

393 test: $\rho=0.026, P=0.45$; Fig. 4). This indicates ongoing gene flow between nearby

394 populations within each ecotype, but amounts of gene flow that do not markedly increase

395 between neighbouring erect and decumbent populations. Intermediate levels of genetic

396 differentiation are present between pairs of erect and decumbent populations from Zambia

397 and Tanzania (genome-wide average $F_{\text {Sт }}: 0.399-0.568$ ), which appears to result from many

398 loci spread across the genome (Fig. S7). 
400 Gene flow between the two ecotypes, and between A. angusta and A. semialata

401 The ABBA-BABA tests revealed extensive gene flow between the erect and decumbent

402 forms of $A$. angusta (Fig. 5). When the least often introgressed erect accession was put in the

403 P1 position (TAN1; Fig. 3B), all tests with an erect in the $\mathrm{P} 2$ position and a decumbent in the

404 P3 position were significant (Fig. 5A). Conversely, when the least often introgressed

405 decumbent accession was put in the P1 position (UGA1; Fig. 5B), only some of the tests with

406 a decumbent in the P2 position and an erect in the P3 position were significant (Fig. 5A). The

407 highest $D$-statistics were observed between the erect ZAM2074-15 and the decumbent

408 ZAM2075-04. Because other accessions from each group also showed elevated $D$-statistics

409 with these two individuals, the exchanges were likely bidirectional. Estimates of admixture

410 proportions using population-level data also support introgression between ecotypes in these

411 two populations (Fig. 3C). In addition, the erect COD1 likely received genes from an

412 unsampled decumbent lineage, explaining that it appears similarly introgressed by all

413 decumbent individuals, and the lineages leading to the erect TAN1601-51 and the decumbent

414 UGA4 likely exchanged genes (Fig. 5A). Other exchanges are difficult to pinpoint precisely,

415 but significant comparisons involving two closely related decumbent (ZAM1930-17 and

416 ZAM1950-10) and two or three of the closely-related erect individuals (ZAM1720-04,

417 ZAM1933-01 and ZAM1930-JKO0102) suggest either exchanges among their common

418 ancestors or repeated exchanges among each of them (Fig. 5A). Importantly, there is no

419 strong evidence of exchanges specifically between the erect and decumbent individuals from

420 the same location with very similar chloroplast genomes (ZAM1930-JKO0102 and

421 ZAM1930-17; Fig. 5A).

ABBA-BABA tests were also conducted to test for introgression between $A$. angusta

423 and its sister species $A$. semialata. When compared to the least often introgressed $A$. angusta 
424 from Uganda (UGA1; Fig. 5C), D-statistics revealed a significant excess of shared derived

425 alleles between all A. semialata individuals and seven of the thirteen A. angusta (Fig. 5D).

426 With the exception of ZAM2074-15, all erect individuals showed significant introgression

427 from A. semialata, while none was detected in the decumbent individuals (except for one of

428 the comparisons involving ZAM1930-17; Fig. 5D). These results indicate that genetic

429 exchanges happened repeatedly between A. semialata and A. angusta, always involving the

430 erect form of the latter (Fig. 5D), which presents similar ecological niches and growth habits

431 as A. semialata (Fig. 1).

433 Discussion

434 We analysed the genetic structure of two newly identified ecotypes of the grass Alloteropsis angusta; a decumbent ecotype restricted to wetlands and an erect ecotype growing in the

436 miombo woodlands and grasslands of tropical Africa (Figs 1B, 1C, S1). The chloroplast

437 genomes are shared among distinct ecotypes that are geographically close. By contrast, the

438 nuclear genomes of the two ecotypes are deeply divergent, despite evidence of secondary

439 gene flow between them. Population genomics of a densely sampled region confirms that the

440 two ecotypes behave as two independent nuclear groups, with only a few admixture events.

441 These patterns are likely the result of pollen-mediated hybridisation followed by movement

442 of the hybrids into a different habitat, where selection favours the paternal genome. Our work

443 therefore provides empirical evidence for a role of hybridisation in facilitating the dispersal

444 of contrasted ecotypes across a large region of Africa. 
448 Selection maintains two contrasted ecotypes despite gene flow

449 Despite their geographic overlap, the two ecotypes of A. angusta are placed in strongly

450 divergent groups in all analyses of nuclear genomes (Figures 2, 3 and 4). The ancestral state

451 of the species is unknown, but coalescence analyses indicate a higher level of allele sorting at

452 the base of the decumbent group (Fig. 2D). This might indicate that the decumbent lineage

453 emerged from within a large erect clade and then underwent an initial bottleneck. The erect

454 and bulbous habit is shared with $A$. semialata, which could support the presence of these

455 characters in their common ancestor. Independently of the direction of the transition, the

456 emergence of a new growth habit has enabled the colonisation of a new habitat, as the two

457 ecotypes are consistently associated with distinct habitats (Figs S1, S2). The population-level

458 analyses identified cases of admixture between some of the erect and decumbent populations

459 (Fig. 3C), and the ABBA-BABA tests revealed multiple cases of introgression among the two

460 ecotypes (Fig. 5). These results demonstrate that the two groups have recurrently interbred.

461 The marked genetic structure must therefore result from strong habit-specific selection. Our

462 scans identify peaks of differentiation distributed throughout the genome (Fig. S7),

463 suggesting that selection has acted on multiple loci. These regions might be involved in

464 dimensions of the phenotype other than the growth habit, as the contrasted habitats will also

465 differ in their light, water, and nutrient availability. Our analyses therefore suggest selection

466 maintained two different life strategies across a vast area despite gene flow.

467

468 Hybridisation allows co-dispersal of ecotypes

469 Unlike the nuclear genome, chloroplast variants are sorted geographically, independently of

470 the ecotype (Fig. 2B). In particular, decumbent and erect individuals occurring across a

471 miombo-wetland boundary in Zambia share almost identical chloroplasts (population 
472 ZAM1930; Figs 2B, S2). Because of their smaller effective population size, haploid

473 chloroplast genomes are more easily introgressed (Martinsen et al., 2001). Frequent

474 chloroplast sharing might therefore result from hybridisation among geographically close

475 populations, a process usually referred to as cytoplasmic capture and reported in multiple

476 species of trees (e.g. Petit et al., 1997; Thomson et al., 2015; Gryta et al., 2017). However,

477 chloroplast-nuclear discrepancies might be better explained by dispersal dynamics (Petit et

478 al., 2003). In most species, the maternally-inherited chloroplast genomes are transported

479 solely by seeds, while nuclear genomes are transported by both seeds and pollen. As a grass,

480 A. angusta is anemophile and its seeds do not present obvious dispersal mechanisms. The

481 ecological specificity of the two ecotypes, combined with a patchy distribution of each

482 environment at the studied scale, means that suitable habitats for either ecotype might be

483 separated by large distances. In wind-dispersed trees, pollen travels further than seeds

484 (Heuertz et al., 2003; Bittencourt \& Sebbenn, 2007), and pollen-mediated gene flow can

485 occur over large distances in grasses (Busi et al., 2008). In such instances, frequent

486 hybridisation should lead to mixing of the nuclear genomes (Wallace et al., 2011; Aguilée et 487 al., 2016). We observe the opposite here, and suggest that episodic long-distance pollen-

488 mediated hybridisation, followed by strong habitat-specific selection, allows the dispersal of

489 one ecotype via the seeds from the other ecotype, as suggested in some trees (Potts \& Reid,

490 1988; Petit et al., 2003). This dispersal mechanism provides a shortcut to the slower process

491 of colonisation by seed. Previous examples concern morphotypes that grow sympatrically in

492 the same habitat types, but our analyses of $A$. angusta show that a similar process enhances

493 the dispersal capabilities of ecotypes otherwise separated by habitat-specific selection.

494 Following long-distance pollination of one established population by the other ecotype,

495 hybrids would bear the local-type chloroplast with a mixed nuclear genome. Their paternal 
496 alleles adapted to the other habitat might allow its colonisation. Following either crosses

497 among hybrids or further long-distance pollination by the other ecotype, selection would

498 repeatedly favour the alleles corresponding to the new habitat. Over time, this process will

499 result in the combination of nuclear genomes mostly matching the new habitat with

500 chloroplast genomes originating from the other habitat. An ecotype can therefore effectively

501 colonise a suitable habitat by hijacking the seeds from the contrasting ecotype, leaving behind

502 only small traces of hybridisation, as detected in some populations by the introgression tests

503 (Fig. 5). In the population with both decumbent and erect individuals (ZAM1930; Fig. S2),

504 admixture was suggested for a few decumbent individuals, but none of the erect individuals

505 (Fig. 3C). In addition, the two individuals with whole-genome sequencing did not show clear

506 evidence of direct exchanges among them (Fig. 5A). In this case, the hybridisation that

507 allowed the exchange of chloroplast genomes left very little traces in the nuclear genome,

508 showing that the alleles from the other type of habitat can be rapidly purged.

510 suggests that such co-dispersal, potentially coupled with post-dispersal introgression,

511 occurred recurrently. The decumbent type is associated with rivers that massively increase in

512 volume during the rainy season, likely providing ample opportunities for unidirectional long-

513 distance seed transport. Distant decumbent populations might then provide a gateway for

514 colonisation of distant habitats by the erect form, contributing to the overall spread of $A$.

515 angusta. Conversely, hybridisation with the erect form growing in woodlands and grasslands

516 would provide access to unconnected river bodies or suitable sites located upstream. We

517 conclude that hybridisation among ecotypes inhabiting markedly different habitats increases

518 the dispersal potential of $A$. angusta. 
520 Interspecific exchanges increase diversity within A. angusta

521 Besides the two A. angusta ecotypes, the conspecific $A$. semialata is also frequent in the

522 region and in several instances was found growing mixed with erect A. angusta (Table S1).

523 Our clustering analyses provide evidence of admixture between $A$. semialata and $A$. angusta

524 in two individuals, always involving the erect ecotype of the latter (Fig. 3). In addition,

525 ABBA-BABA tests indicate that the erect individuals of A. angusta are significantly more

526 introgressed than their decumbent conspecifics (Fig. 5). The erect form of A. angusta and A.

527 semialata co-occur frequently and share the same habitats, which would increase

528 opportunities for hybridisation. In addition, the similarity of growth forms and habitats might

529 have favoured the sharing of adaptive alleles via selective introgression. Some individuals of

530 A. angusta are very similar to A. semialata (Fig. 1), with large deep bulbs that are not usually

531 found in A. angusta. Genes responsible for such traits might have crossed the species

532 boundaries, as reported for elements of the $\mathrm{C}_{4}$ photosynthetic pathway (Dunning et al., 2017).

533 The two species therefore form a species complex, with recurrent genetic exchanges between

534 the two ecotypes of $A$. angusta (and the different subgroups of A. semialata; Bianconi et al.,

535 2020; Olofsson et al., 2021), but also episodic exchanges among A. semialata and A. angusta.

536 The impact of these exchanges remains speculative, but they might have contributed to the

537 functional and ecological diversity of the group.

\section{Conclusions}

540 In this work, we show that the grass A. angusta exists across tropical Africa as two contrasted

541 ecotypes that correspond to distinct genetic groups. The erect form, previously mistaken for

542 the conspecific $A$. semialata, is widespread in miombo woodlands, while the decumbent form

543 occurs in wetlands bordering lakes and rivers. Despite deep nuclear divergence, we find 
544 evidence of genetic exchanges and different morphs from a given geographical region share

545 chloroplast genomes. These patterns indicate recurrent hybridisation followed by selection

546 that sorts most of the genomes by habitat. These hybridisation events offer opportunities for

547 dispersal to distant locations by effectively hijacking the seeds from the other ecotype. We

548 conclude that hybridisation, coupled with strong selection, can boost plant dispersal without

549 erasing the associations between genomes and environments. A similar mechanism has been

550 previously proposed among tree morphotypes, and we offer here empirical evidence of co-

551 dispersal among grasses adapted to contrasting habitats.

553 Acknowledgements

554 This work was funded by the European Research Council (grant ERC-2014-STG-638333)

555 and the Royal Society (grant RGF\EA $\backslash 181050$ ), and has benefited from "Investissements

556 d'Avenir" grants managed by the Agence Nationale de la Recherche (CEBA, ref. ANR-10-

557 LABX-25-01 and TULIP, ref. ANR-10-LABX-41). P.A.C. is funded by a Royal Society

558 University Research Fellowship (grant URF\R\180022).

559

560 Author contributions

561 EVC, MSS, JKO and PAC designed the study. EVC, JKO, FN, MEB and PAC performed 562 field work. EVC, MSS, JKO, GS, SM, and GB produced the genomic data. EVC analysed the 563 data, with the help of MSS, JKO, MEB, and PAC. EVC and PAC wrote the paper, with the 564 help of all authors. 
568

569 Sequence data have been deposited in the NCBI Sequence Read Archive with the project

570 number PRJNA715711. Scripts for analysis and data processing available at:

571 https://github.com/evcurran/Angusta-Pop-Genomics

\section{References}

574 Abbott R, Albach D, Ansell S, Arntzen JW, Baird SJE, Bierne N, Bougham J, Brelsford A, Buerkle CA, Buggs R et al. 2013. Hybridization and speciation. Journal of Evolutionary Biology 26: 229-246.

Aguilée R, Raoul G, Rousset F, Ronce O. 2016. Pollen dispersal slows geographical range shift and accelerates ecological niche shift under climate change. Proceedings of the

$$
\text { L. 2016. Borrowed alleles and convergence in serpentine adaptation. Proceedings of }
$$
the National Academy of Sciences of the United States of America, 113: 8320-8325.

583 Barton NH, Hewitt GM. 1985. Analysis of hybrid zones. Annual Review of Ecology and Systematics, 16: 113-148.

Bhatia G, Patterson N, Sankararaman S, Price AL. 2013. Estimating and interpreting $F_{\text {ST: }}$ The impact of rare variants. Genome Research 23: 1514-1521.

587 Bianconi ME, Dunning LT, Curran EV, Hidalgo O, Powell RF, Mian S, Leitch IJ, Lundgren MR, Manzi S, Vorontsova MS, et al. Contrasted histories of organelle and nuclear genomes underlying physiological diversification in a grass species. Proceedings of the Royal Society B: Biological Sciences 287: 20201960. https://doi.org/10.1098/rspb.2020.1960. 
592 Bittencourt JVM, Sebbenn AM. 2007. Patterns of pollen and seed dispersal in a small, fragmented population of the wind-pollinated tree Araucaria angustifolia in southern Brazil. Heredity 99: 580-591.

Bolger AM, Lohse M, Usadel B. 2014. Trimmomatic: A flexible trimmer for Illumina sequence data. Bioinformatics 30: 2114-2120.

597 Broad Institute. 2019. Picard Toolkit. GitHub Repository. http://broadinstitute.github.io/picard/; Broad Institute

599 Busi R, Yu Q, Barrett-Lennard R, Powles S. 2008. Long distance pollen-mediated flow of herbicide resistance genes in Lolium rigidum. Theoretical and Applied Genetics 117:

602 Capella-Gutiérrez S, Silla-Martínez JM, Gabaldón T. 2009. trimAl: A tool for automated alignment trimming in large-scale phylogenetic analyses. Bioinformatics 25: 19721973.

605 Catchen J, Hohenlohe PA, Bassham S, Amores A, Cresko WA. 2013. Stacks: An analysis tool set for population genomics. Molecular Ecology 22: 3124-3140.

607 Clayton WD, Renvoize SA. 1982. Flora of tropical East Africa. Gramineae. Part 3. AA Balkema, Rotterdam.

609 Drummond AJ, Rambaut A. 2007) BEAST: Bayesian evolutionary analysis by sampling trees. BMC Evolutionary Biology 7: 214.

611 Dunning LT, Lundgren MR, Moreno-Villena JJ, Namaganda M, Edwards EJ, Nosil P, Osborne CP, Christin PA. 2017. Introgression and repeated co-option facilitated the recurrent emergence of $\mathrm{C}_{4}$ photosynthesis among close relatives. Evolution 71: 15411555.

615 Dunning LT, Olofsson JK, Parisod C, Choudhury RR, Moreno-Villena JJ, Yang Y, Dionora 
J, Quick PW, Park M, Bennetzen JL et al. 2019. Lateral transfers of large DNA fragments spread functional genes among grasses. Proceedings of the National Academy of Sciences of the United States of America 116: 4416-4425.

Durand EY, Patterson N, Reich D, Slatkin M. 2011. Testing for ancient admixture between closely related populations. Molecular Biology and Evolution 28: 2239-2252.

Ellstrand NC. 1992. Gene flow by pollen: Implications for plant conservation genetics. Oikos 63: 77.

Ellstrand NC. 2014. Is gene flow the most important evolutionary force in plants? American Journal of Botany 101: 737-753.

Evanno G, Regnaut S, Goudet J. 2005. Detecting the number of clusters of individuals using the software STRUCTURE: A simulation study. Molecular Ecology 14: 2611-2620.

Galindo J, Martínez-Fernández M, Rodríguez-Ramilo ST, Rolán-Alvarez E. 2013. The role of local ecology during hybridization at the initial stages of ecological speciation in a marine snail. Journal of Evolutionary Biology 26: 1472-1487.

Green RE, Krause J, Briggs AW, Maricic T, Stenzel U, Kircher M, Patterson N, Li H, Zhai W, Fritz MHS, et al. 2010. A draft sequence of the neandertal genome. Science 328: $710-722$.

Grass Phylogeny Working Group. 2001. Phylogeny and subfamilial classification of the grasses (Poaceae). Annals of the Missouri Botanical Garden 88: 373-457.

Gryta H, Van de Paer C, Manzi S, Holota H, Roy M, Besnard G. 2017. Genome skimming and plastid microsatellite profiling of alder trees (Alnus spp., Betulaceae): phylogenetic and phylogeographical prospects. Tree Genetics and Genomes 13: 118.

Hall RJ. 2016. Hybridization helps colonizers become conquerors. Proceedings of the National Academy of Sciences of the United States of America 113: 9963-9964. 
640 Heuertz M, Vekemans X, Hausman JF, Palada M, Hardy OJ. 2003. Estimating seed vs. pollen dispersal from spatial genetic structure in the common ash. Molecular Ecology 12: $2483-2495$.

Hudson RR, Slatkin M, Maddison WP. 1992. Estimation of levels of gene flow from DNA sequence data. Genetics 132: 583-589. frost and drought. Plant, Cell \& Environment 31: 1038-1050. https://doi.org/10.1111/j.1365-3040.2008.01815.x

649 Kellogg EA. 2015. Flowering Plants. Monocots: Poaceae. Heidelberg: Springer. p. 416.

650 Kopelman NM, Mayzel J, Jakobsson M, Rosenberg NA, Mayrose I. 2015. Clumpak: A program for identifying clustering modes and packaging population structure inferences across K. Molecular Ecology Resources 15: 1179-1191.

653 Korneliussen TS, Albrechtsen A, Nielsen R. 2014. ANGSD: Analysis of next generation sequencing data. BMC Bioinformatics 15: 1-13.

655 Langmead B, Salzberg SL. 2012. Fast gapped-read alignment with Bowtie 2. Nature Methods 9: 357-359.

Li H. 2011. A statistical framework for SNP calling, mutation discovery, association mapping and population genetical parameter estimation from sequencing data. Bioinformatics, 27: 2987-2993. 

the niche within a single species. Ecology Letters 18: 1021-1029.

Martinsen GD, Whitham TG, Turek RJ, Keim P. 2001. Hybrid populations selectively filter gene introgression between species. Evolution 55: 1325-1335.

Meisner J, Albrechtsen A. 2018. Inferring population structure and admixture proportions in low-depth NGS data. Genetics 210: 719-731.

Nychka D, Furrer R, Paige J, Sain S. 2017. “fields: Tools for spatial data.” doi:

\subsection{5/D6W957CT}

O’Connell J, Schulz-Trieglaff O, Carlson E, Hims MM, Gormley NA, Cox AJ. 2015. 2037.

678 Olofsson JK, Bianconi M, Besnard G, Dunning LT, Lundgren MR, Holota H, Vorontsova MS, Hidalgo O, Leitch IJ, Nosil P, et al. 2016. Genome biogeography reveals the intraspecific spread of adaptive mutations for a complex trait. Molecular Ecology 25:

682 Olofsson JK, Dunning LT, Lundgren MR, Barton HJ, Thompson J, Cuff N, Anyarathne M, 
688

689

690

in a grass maintain photosynthetic diversity despite gene flow and habitat overlap. Molecular Ecology https://doi.org/10.1111/mec.15871

Patel RK, Jain M. 2012. NGS QC toolkit: A toolkit for quality control of next generation sequencing data. PLoS ONE 7: e30619.

Perreta M, Ramos J, Tivano JC, Vegetti A. 2011. Descriptive characters of growth form in Poaceae - An overview. Flora: Morphology, Distribution, Functional Ecology of Plants 206: 283-293.

Petit RJ, Bodénès C, Ducousso A, Roussel G, Kremer A. 2003. Hybridization as a mechanism of invasion in oaks. New Phytologist 161: 151-164.

Petit RJ, Pineau E, Demesure B, Bacilieri R, Ducousso A, Kremer A. 1997. Chloroplast DNA footprints of postglacial recolonization by oaks. Proceedings of the National Academy of Sciences of the United States of America 94: 9996-10001.

Potts BM, Reid JB. 1988. Hybridization as a dispersal mechanism. Evolution 42: 1245-1255.

R Core Team. 2019. R: A language and environment for statistical computing. R Foundation for Statistical Computing, Vienna, Austria. URL https://www.R-project.org/

Rambaut A, Suchard MA, Xie W, Drummond AJ. 2013. Tracer v1.6. Available from: http://tree.bio.ed.ac.uk/software/tracer/

Schluter D. 2001. Ecology and the origin of species. Trends in Ecology \& Evolution 16: 372380.

Schneider CA, Rasband WS, Eliceiri KW. 2012. NIH Image to ImageJ: 25 years of image analysis. Nature Methods 9: 671-675.

Seehausen O, Butlin RK, Keller I, Wagner CE, Boughman JW, Hohenlohe PA, Peichel CL, Saetre GP, Bank C, Brännström Å, et al. 2014. Genomics and the origin of species. Nature Reviews Genetics 15: 176-192. 
712

713

714

715

716

717

718

719

720

Skotte L, Korneliussen TS, Albrechtsen A. 2013. Estimating individual admixture proportions from next generation sequencing data. Genetics 195: 693-702.

Song Y, Endepols S, Klemann N, Richter D, Matuschka FR, Shih CH, Nachman MW, Kohn MH. 2011. Adaptive introgression of anticoagulant rodent poison resistance by hybridization between old world mice. Current Biology 21: 1296-1301.

Soria-Carrasco V, Gompert Z, Comeault AA, Farkas TE, Parchman TL, Johnston JS, Buerkle CA, Feder JL, Bast J, Schwander T, et al. 2014. Stick insect genomes reveal natural selection's role in parallel speciation. Science 344: 738-742.

Stamatakis A. 2014. RAxML version 8: A tool for phylogenetic analysis and post-analysis of large phylogenies. Bioinformatics 30: 1312-1313.

Stankowski S, Sobel JM, Streisfeld MA. 2017. Geographic cline analysis as a tool for studying genome-wide variation: a case study of pollinator-mediated divergence in a monkeyflower. Molecular Ecology 26: 107-122.

Stapf O. 1919. In Daniel, O. 1919. Flora of Tropical Africa. Crown Agents for Overseas Governments and Administrations, London. pp. 482-486.

Stent SM, Rattray JM. 1933. The grasses of southern Rhodesia. Proceedings and Transactions of the Rhodesia Scientific Association. Bulawayo 32: 21-22.

Taylor EB, Boughman JW, Groenenboom M, Sniatynski M, Schluter D, Gow JL. 2006. Speciation in reverse: Morphological and genetic evidence of the collapse of a threespined stickleback (Gasterosteus aculeatus) species pair. Molecular Ecology 15: 343355.

The Heliconius Genome Consortium. 2012. Butterfly genome reveals promiscuous exchange of mimicry adaptations among species. Nature 487: 94-98.

Thomson AM, Dick CW, Pascoini AL, Dayanandan S. 2015. Despite introgressive 
hybridization, North American birches (Betula spp.) maintain strong differentiation at nuclear microsatellite loci. Tree Genetics and Genomes, 11: 101.

738 Todesco M, Pascual MA, Owens GL, Ostevik KL, Moyers BT, Hübner S, Heredia SM, Hahn MA, Caseys C, Bock DG, Rieseberg LH. 2016. Hybridization and extinction. Evolutionary Applications 9: 892-908.

741 Wallace LE, Culley TM, Weller SG, Sakai AK, Kuenzi A, Roy T, Wagner WL, Nepokroeff M. 2011. Asymmetrical gene flow in a hybrid zone of Hawaiian Schiedea

743 (Caryophyllaceae) species with contrasting mating systems. PLoS ONE 6: 1-12.

744 Zhang C, Rabiee M, Sayyari E, Mirarab S. 2018. ASTRAL-III: Polynomial time species tree reconstruction from partially resolved gene trees. BMC Bioinformatics 19: 15-30. 


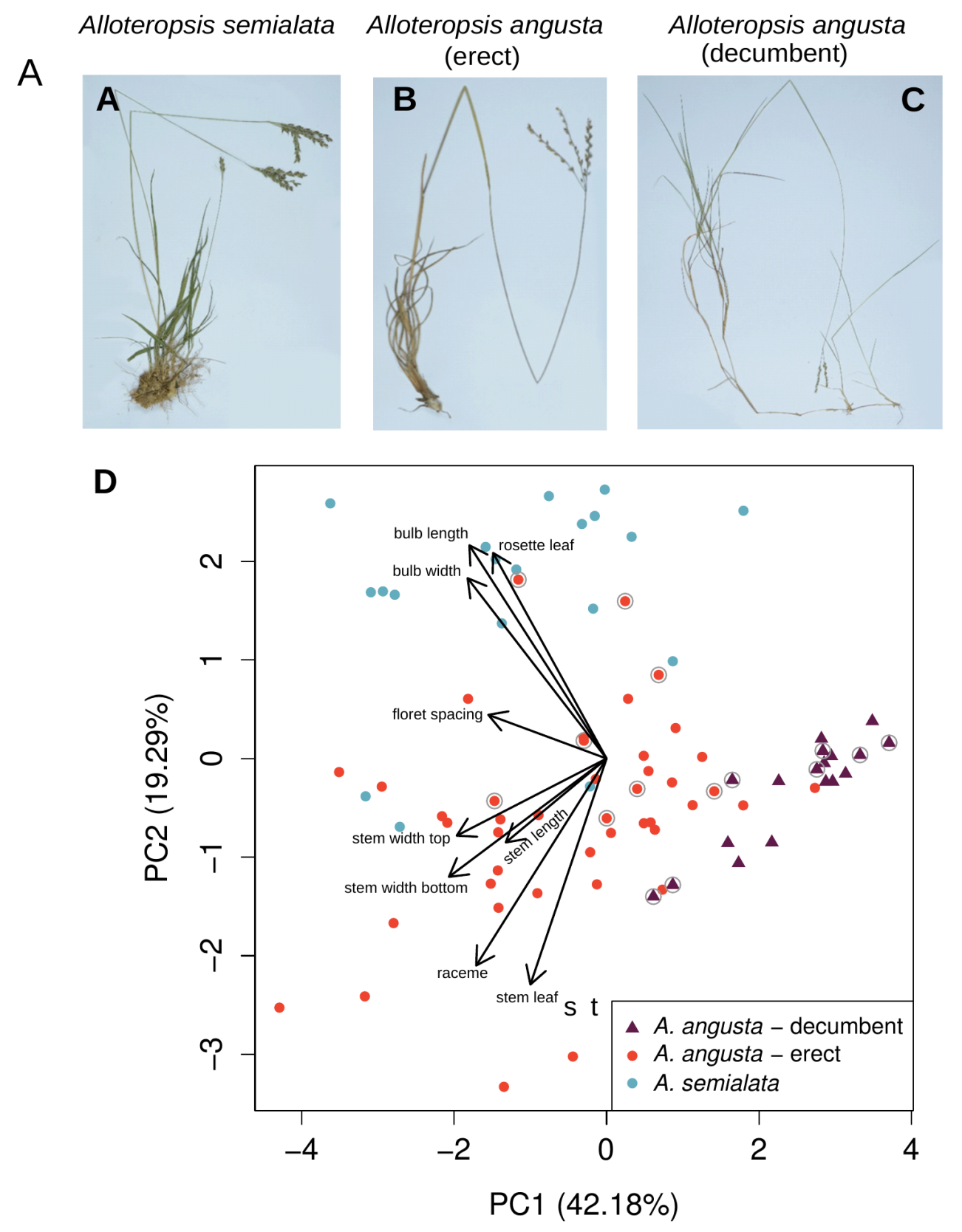

746 Figure 1. Morphological variation within Alloteropsis angusta and its sister species A. semialata.

747 Digitised herbarium specimens of A) A. semialata [ZAM1503 - Nyirenda, Lundgren Dunning 3

748 (SHD)], B) A. angusta - erect ecotype [TAN1601 - Dunning, Dunning, Kayombo 1 (SHD)], and C)

749 A. angusta - decumbent ecotype [M. R. Lundgren 2015-3-3 (SHD)]. D) Morphological variation

750 among A. semialata and A. angusta, as assessed by the first two axes of a principal component

751 analysis. Contributions of the different variables are indicated with arrows. Individuals with a grey

752 circle are included in the whole-genome phylogeny. 
A

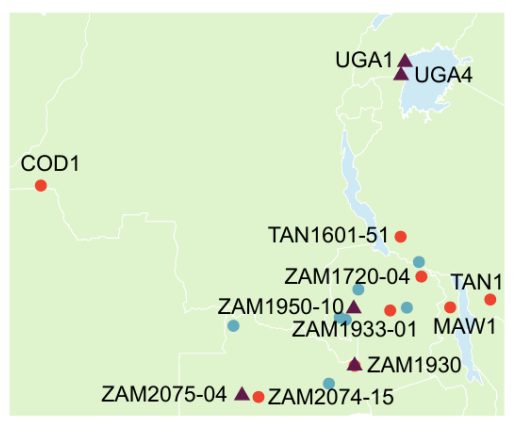

C

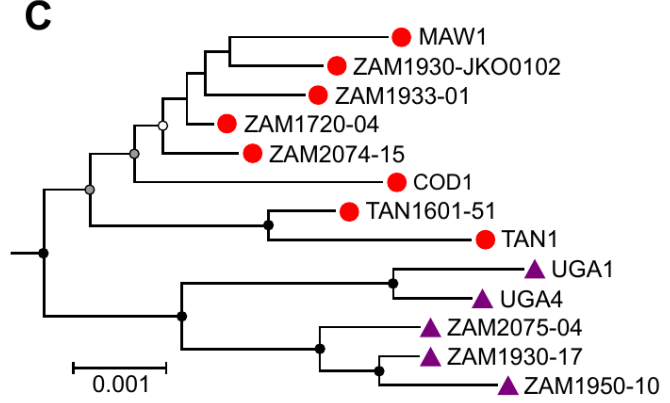

B

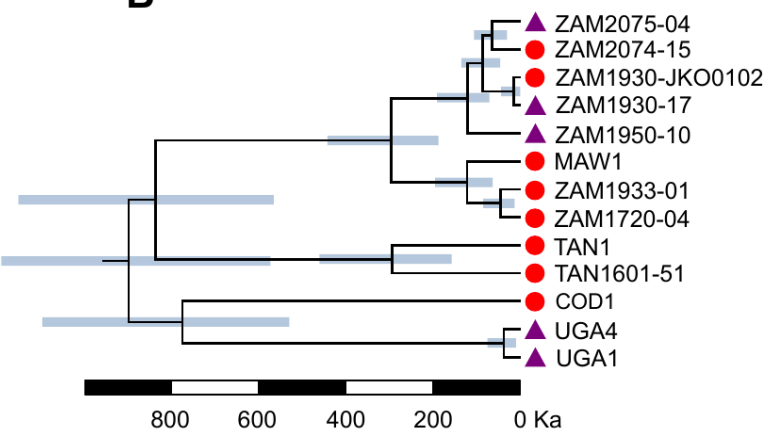

D

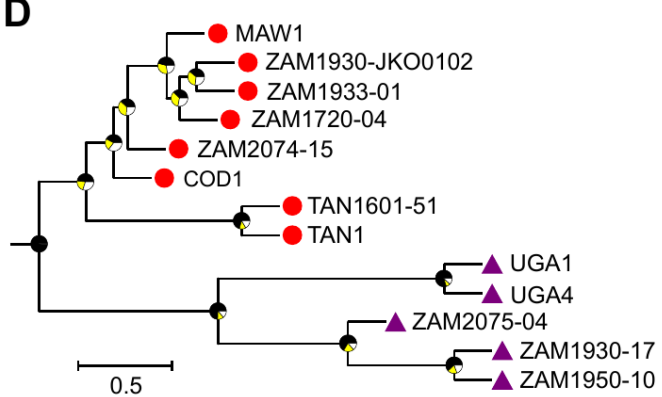

753 Figure 2. Phylogenetic relationships among Alloteropsis angusta samples.

754 A) The geographic distribution of individuals of Alloteropsis angusta and A. semialata included in the

755 phylogenetic tree is shown. Erect individuals of $A$. angusta are shown with red circles, and decumbent

756 individuals with purple triangles. Samples of A. semialata are shown with blue circles. B) The portion

757 of the time-calibrated phylogenetic tree corresponding to A. angusta is shown (see Fig. S3 for full

758 tree). Bars at nodes indicate $95 \%$-confidence intervals. All nodes had support values of 1.0. The scale

759 is given in thousands of years (Ka). C) The portion of the maximum-likelihood tree based on nuclear

760 variants corresponding to A. angusta is shown (see Fig. S3 for full tree). Dots on nodes show

761 bootstrap support values; $100=$ black, $>90=$ grey, $>60=$ white. D) The portion of the multigene

762 coalescence species tree corresponding to A. angusta is shown (see Fig. S4 for full tree). Pie charts on

763 nodes show the proportions of quartets supporting the main topology (in black) and the two

764 alternatives. All nodes have support values above 0.97 . The scale is given in coalescence units.

765 Terminal branches are given arbitrary lengths. The scale is given in expected substitutions per site.

766 For B), C), and D), ecotypes are shown at tips; red circles = erect individuals, purple triangles =

767 decumbent individuals. 
A

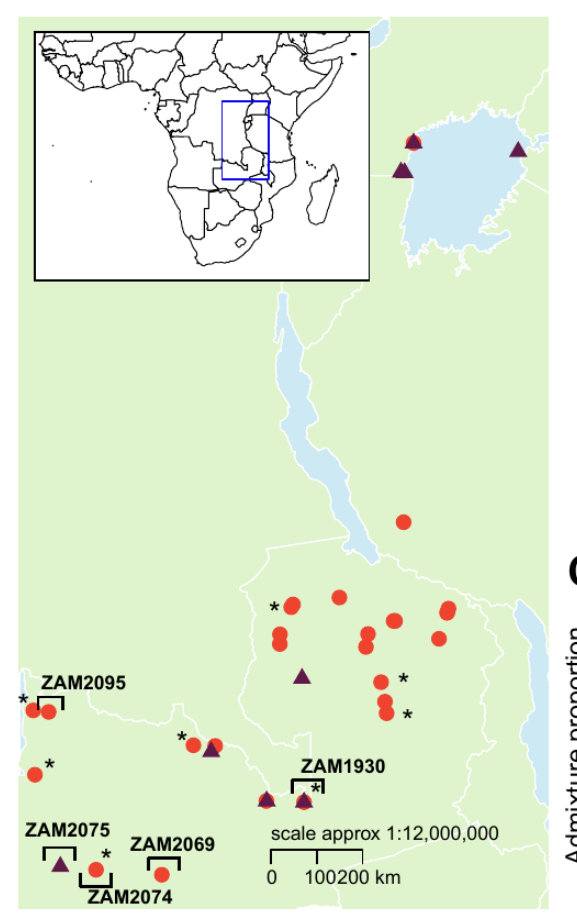

B

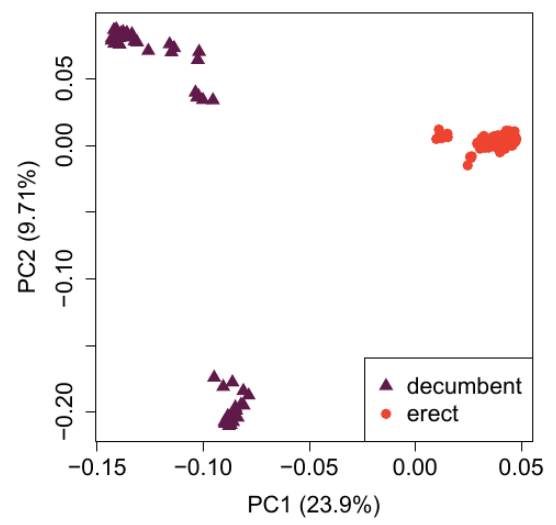

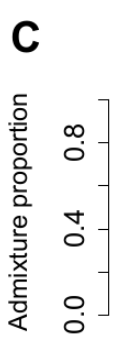

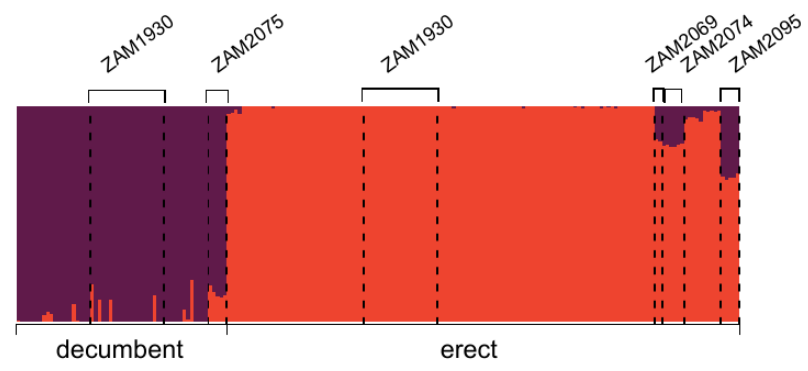

768 Figure 3. Population structure of Alloteropsis angusta in Zambia, Tanzania and Uganda.

769 A) Geographic distribution of populations of A. angusta which were sampled and genotyped using

770 RAD-sequencing. Red circles indicate erect populations, while decumbent populations are shown

771 with purple triangles. Populations highlighted with an asterisk were sympatric with A. semialata. B)

772 Principal component analysis of genetic variation among all RAD-sequenced A. angusta samples. C)

773 Results of an admixture analysis at $K=2$. Each vertical bar represents one individual. The major

774 genetic groups are delimited at the bottom. Populations of interest are delimited by dashed vertical

775 lines, with names at the top. 


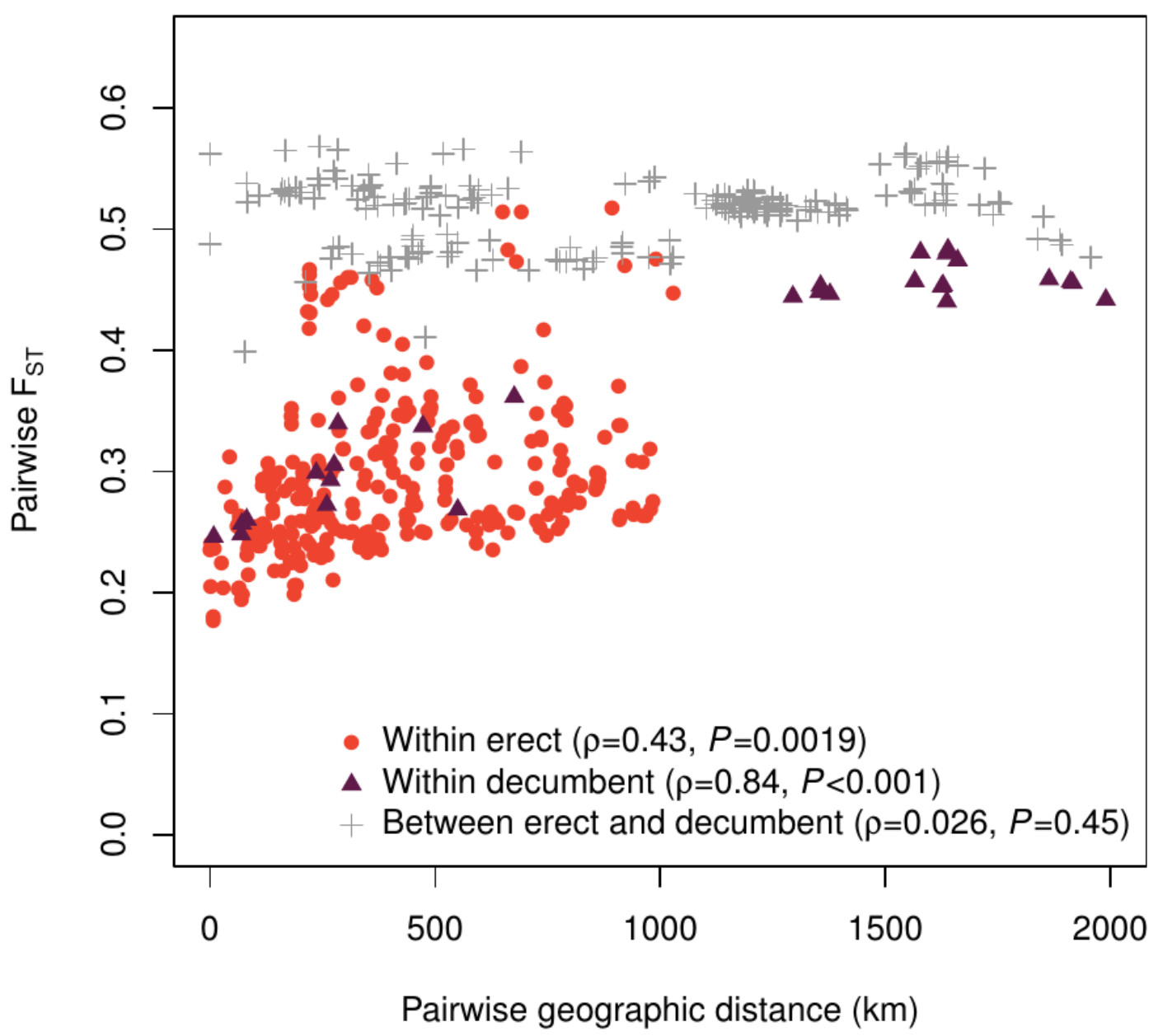

783 Figure 4. Patterns of isolation-by-distance within and between the two ecotypes of Alloteropsis angusta.

785 Pairwise genetic differentiations $\left(F_{\mathrm{ST}}\right)$ among all pairs of populations are plotted against geographic

786 distances $(\mathrm{km})$. Red circles indicate comparisons between erect populations, purple triangles indicate

787 comparisons between decumbent populations, and grey crosses indicate comparisons between

788 contrasting ecotypes. For each group, the Spearman correlation coefficient is indicated, with the $p$ -

789 value from a Mantel test. 


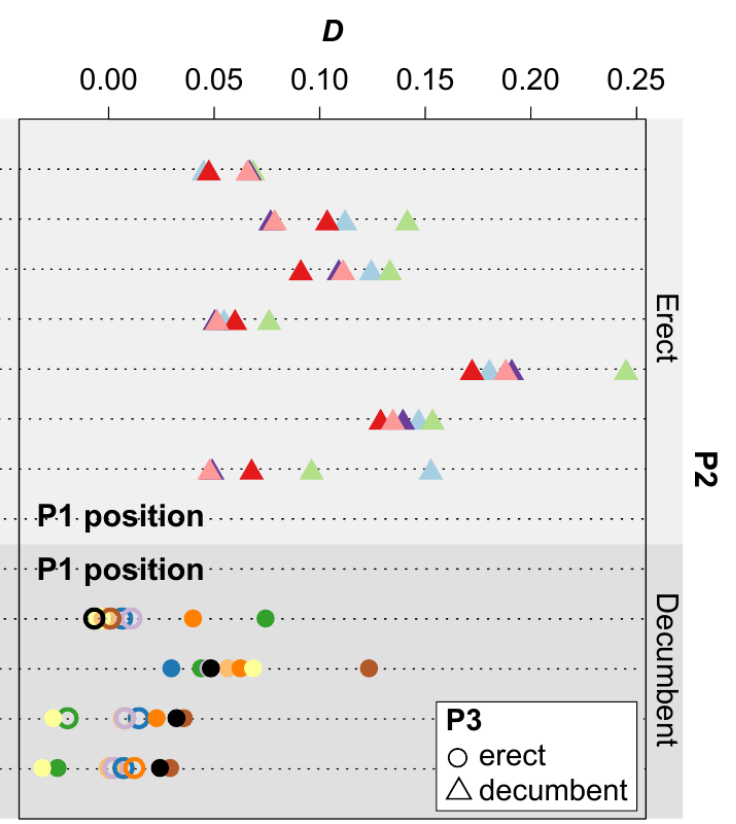

B
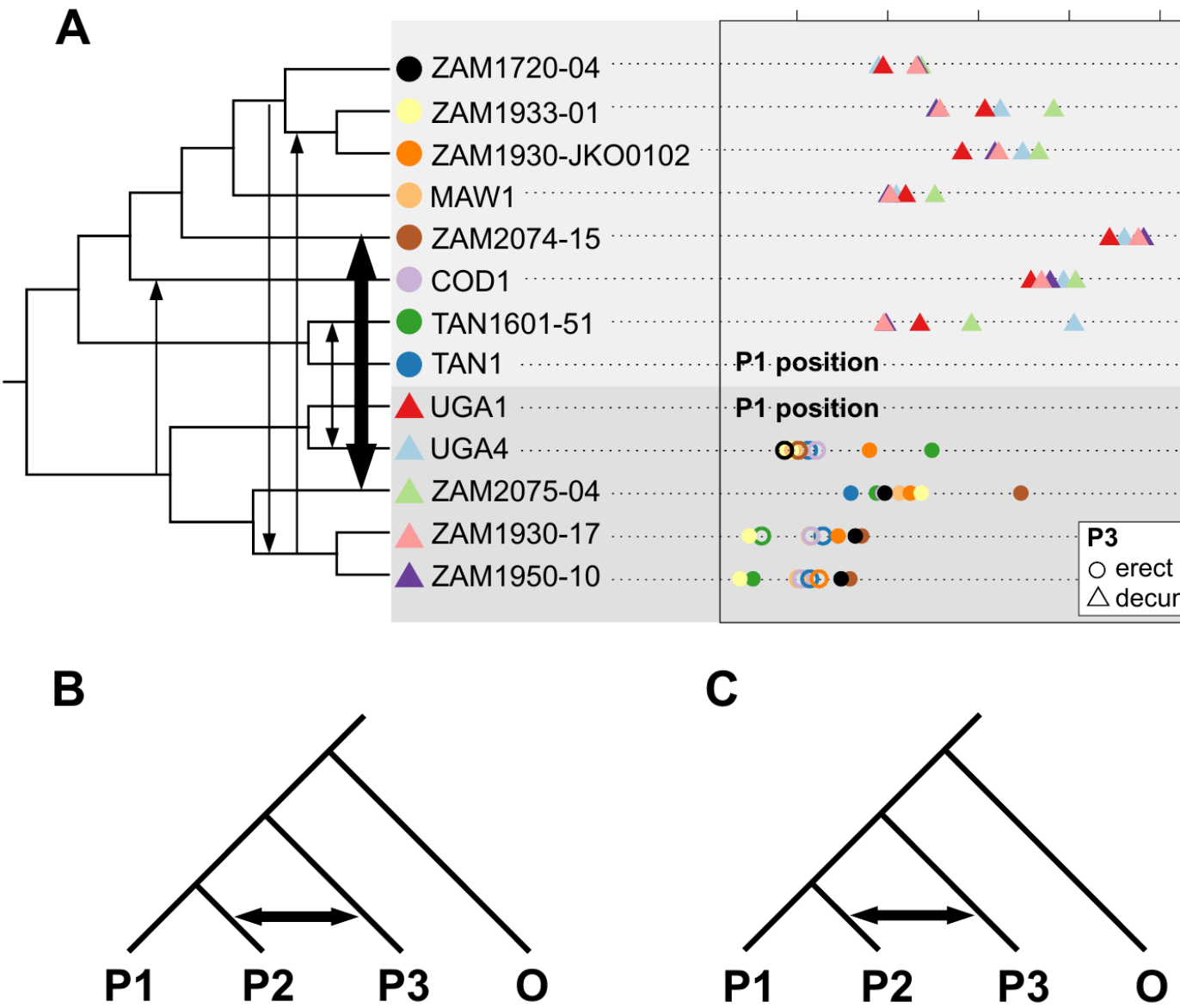

TAN1 erect dec cim

UGA1 dec erect cim

UGA1 ang sem cim
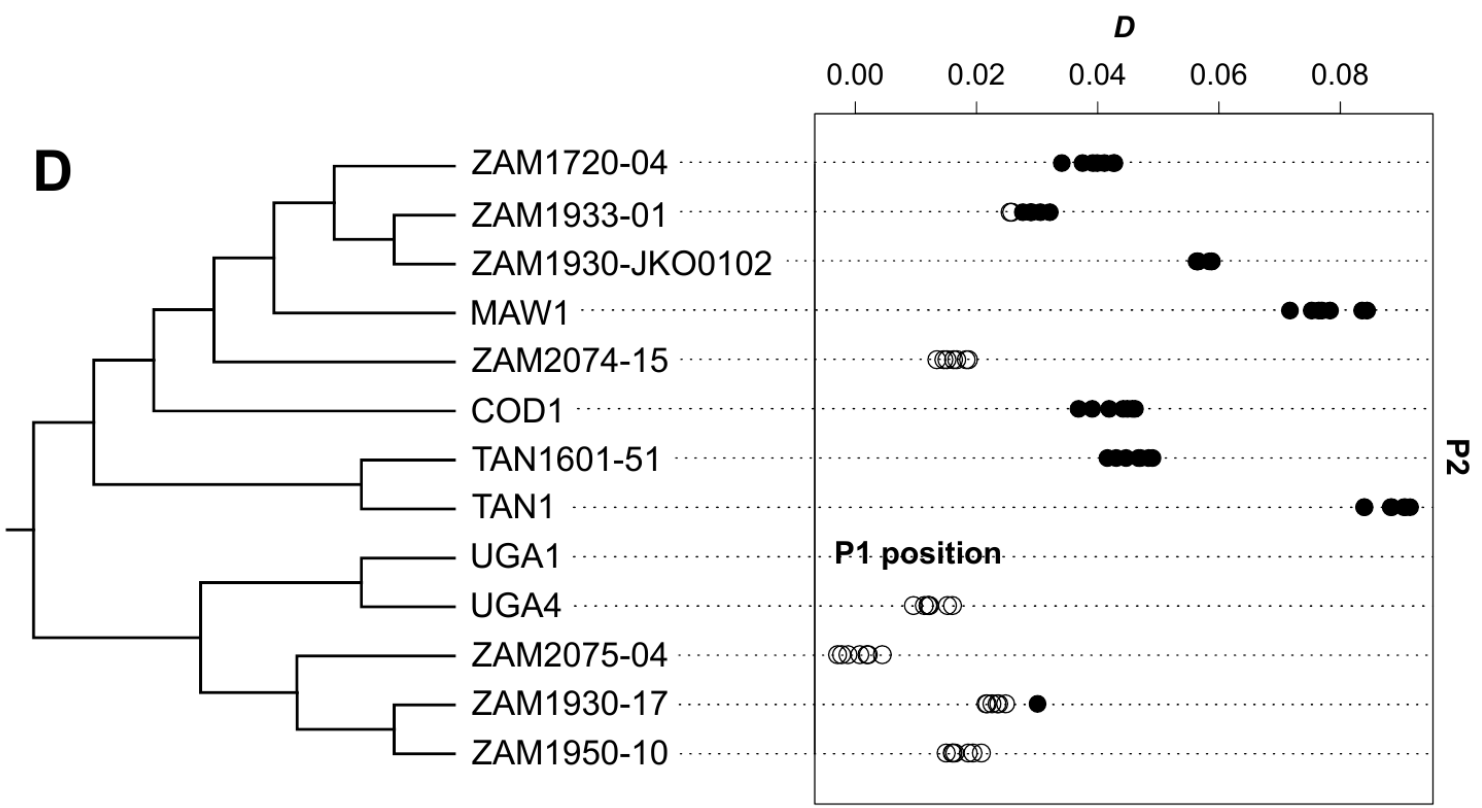
796 Figure 5. Evidence of introgression in Alloteropsis angusta.

797 A) Distribution of $D$-statistics for each individual in the position P2 (ordered as in the multigene

798 coalescence tree; Figure 2D), when the P1 position was occupied by the least often introgressed

799 individual from the same ecotype and the P3 position was occupied by an individual from the other

800 ecotype. The identity of the individual in the P3 position is indicated with symbols that match those in

801 front of the phylogeny. Empty symbols show non-significant tests, filled symbols show significant

802 tests. Arrows on the phylogenetic tree show the main genetic exchanges suggested by the $D$-statistics.

803 B) Configuration used to detect introgression among the two ecotypes of A. angusta, with generic

804 names of the positions (first line), the configuration focused on the erect individuals (second line), and

805 the configuration focused on the decumbent individuals (third line; dec $=$ decumbent, $\operatorname{cim}=A$.

806 cimicina). The arrow shows exchanges indicated by a positive $D$-statistic. C) Configuration used to

807 detect introgression between A. angusta and A. semialata, using the same structure as in panel $\mathbf{B}$ (ang

$808=$ A. angusta , sem = A. semialata . . D) Distribution of $D$-statistics for each A. angusta individual in the

809 P2 position (least often introgressed A. angusta individual in P1 position, A. semialata in P3 position;

810 see structure in panel $\mathbf{C}$ ). Individuals are arranged as in the multigene coalescence species tree (see

811 Figure 2D). Empty circles indicate non-significant tests, with significant tests shown with filled

812 circles. 